\title{
RECIPROCAL BEATING INITIATED BY VENTRICULAR PREMATURE SYSTOLES
}

BY

\author{
R. LANGENDORF, L. N. KATZ, AND A. J. SIMON * \\ From the Cardiovascular Department of Michael Reese Hospital, Chicago, Ill.
}

Received August 12, 1943

Reciprocal rhythm is a rare phenomenon. All reported cases with but one exception are instances of nodal rhythm with re-entry of the retrograde nodal impulse $(1-3,5-10,12,15$, \& 21-24). However, Wolferth and McMillan (1939) have described a case of sinus rhythm with re-entry of the antegrade sinus impulse. In the present communication a unique case is reported in which the reciprocal rhythm was initiated by ventricular premature systoles. So far as we are aware, this has not been described before in a human heart although it has been produced in the experimental animal $(14,17, \& 19)$. The patient manifesting this arrhythmia was a 56 year old white woman (a private patient of Dr. D. C. Straus) who presented no evidence of organic heart disease and was sent up for analysis of her pulse irregularity. In Fig. 1 is shown her first cardiogram and in Fig. 2 records taken during and after left carotid sinus pressure and after exercise.

Fig. 1 shows a group of four ventricular beats which repeat themselves throughout, except at the end of lead III. The four QRS complexes in each group will be called $R_{d}, R_{e}, R_{f}$, and $R_{g}$ respectively, and the three $P$ waves, $P_{a}, P_{b}$, and $P_{c}$ respectively.

It will be seen that $P_{a}$ and $P_{b}$ precede $R_{d}$ and $R_{e}$ and that the $P_{a}-R_{d}$ and $P_{b}-R_{e}$ intervals are constant in each lead and measure $0.14 \mathrm{sec}$. in lead II $(0.08 \mathrm{sec}$. when measured from the peak of $\mathrm{P}$ to QRS onset). Further, the $\mathrm{P}_{\mathrm{a}}-\mathrm{P}_{\mathrm{b}}$ intervals vary slightly; in lead I from 0.92-0.94 sec., in lead II from 0.90-0.94 sec., in lead III from 0.86-0.90 sec., and in the chest leads from 0.90-0.92 sec. The mechanism for the first two beats of the groups of four is therefore a sinus arrhythmia varying in rate from 64 to 70 beats per minute.

The third ventricular beat, $R_{f}$, is obviously a premature ventricular systole since it is premature, is not preceded by a $P$ wave, and is prolonged and bizarre compared to $\mathbf{R}_{d}$ and $\mathbf{R}_{\mathbf{e}}$.

The fourth ventricular beat, $R_{g}$, is preceded by a $P$ wave, $P_{c}$, in every instance, and it resembles $\mathbf{R}_{d}$ and $R_{e}$ closely. Three types of $P_{c}$ are seen:

(a) one that resembles $\mathrm{P}_{\mathrm{a}}$ and $\mathrm{P}_{\mathrm{b}}$ making allowances for the fact that $\mathrm{P}_{\mathrm{c}}$ occurs superimposed on the S-T-T complex of $R_{f}$, viz. the second $P_{c}$ in $(C)$, the third $P_{c}$ in (D), and probably all the $\mathrm{P}_{\mathrm{c}}$ waves in $(\mathrm{A})$;

(b) one that is strikingly different from $P_{a}$ and $P_{b}$. This is best seen in leads II and III where it is sharply inverted while $P_{a}$ and $P_{b}$ are upright, viz. the second $P_{c}$ in (B) and the first $P_{c}$ in (D);

and $(c)$ one that is intermediate in contour between $(a)$ and $(b)$, viz. the first and third $P_{c}$ in (B), the first and third $P_{c}$ in (C), and the second and fourth $P_{c}$ in (D).

All three types of $P_{c}$ are characterized by a tendency towards prematurity, viz. $\mathbf{P}_{b}-\mathbf{P}_{c}$ is shorter than $P_{a}-P_{b}$. The $P_{c}-R_{g}$ interval and the $R_{t}-P_{c}$ intervals are not easily measured because the beginning of $P_{c}$ cannot be located exactly. However, by comparing the intervals

* Aided by the A. D. Nast Fund for Cardiovascular Research. The department is supported in part by the Michael Reese Research Foundation. 


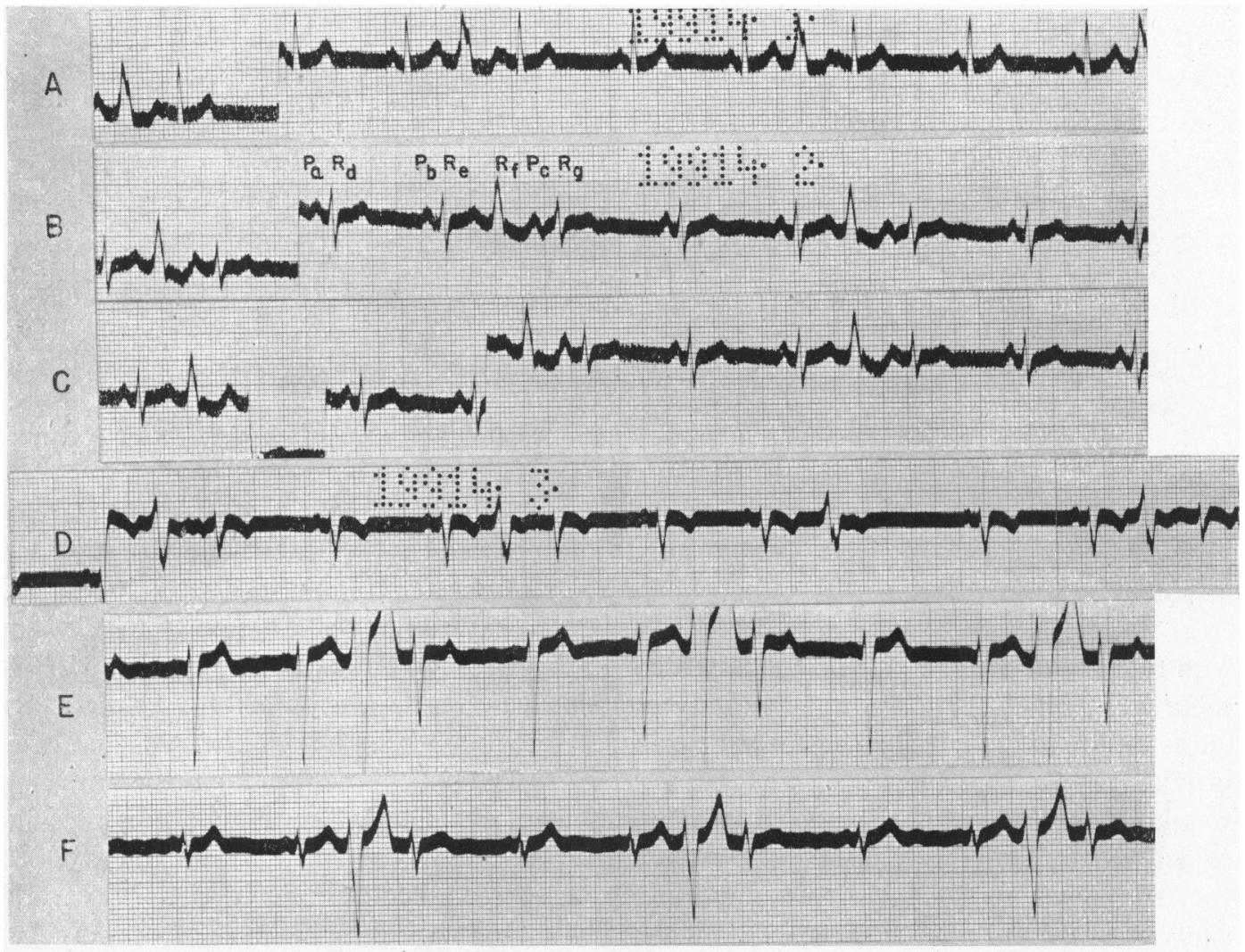

FIG. 1.-Electrocardiogram from the patient at his first visit. (A) is lead I; (B) and (C) are a continuous strip of lead II, the last beat of (B) being repeated as the first beat of (C). In (B) a group $\mathbf{P}_{a}, \mathbf{R}_{d}, \mathbf{P}_{b}, \mathbf{R}_{e}^{\mathbb{E}}, \mathbf{R}_{\mathfrak{f}}, \mathbf{P}_{\mathrm{c}}, \mathbf{R}_{\mathrm{g}}$ is labelled. Discussed in text. (D), (E), and (F) are respectively leads III, $\mathrm{CF}_{2}$, and $\mathrm{CF}_{4}$. Note that $\mathbf{P}_{\mathrm{c}}$ in the chest leads (E and $F$ ) is difficult to make out.

measured from the peak of $P_{c}$ with similar measurements made from the peak of $P_{a}$ and $P_{b}$, it is apparent that $P_{c}-R_{g}\left(0.08-0.16\right.$ sec.) is equal to or longer than $P_{a}-P_{d}$ and $P_{b}-R_{e}$. The $R_{f}-R_{g}$ interval is constant for each lead, regardless of the position and contour of the intervening $P_{c}$. In leads I and the chest leads it equals $0.50 \mathrm{sec}$. and in leads II and III, $0.52 \mathrm{sec}$. Likewise such measurements show that $R_{f}-P_{c}$ is equal to about $0.28-0.44$ sec.; in those instances where $P_{c}$ is inyerted $R_{f}-P_{c}$ is constant and measures 0.44 sec., and the same value is obtained in the third $P_{c}$ in lead II where $P_{c}$ is intermediate in contour between $P_{a}$ and the inverted $P_{c}$; in all other instances $R_{f}-P_{c}$ is shorter than $0.44 \mathrm{sec}$. and the contour of $P_{c}$ varies.

On one occasion in lead III, $P_{c}$ is not followed by $R_{g}$. In this latter instance $P_{c}$ resembles $P_{a}$ and $P_{b}$, and $R_{f}-P_{c}$ is equal to 0.28 sec. $R_{f}$ occurred later than usual in this instance, viz. $R_{e}-R_{f}$ here equals 0.52 sec. as compared with the usual value of $0.41-0.44$ sec. in the limb leads and $0.48 \mathrm{sec}$. in the chest leads.

At first sight the beat $\mathbf{P}_{c} R_{g}$ might be considered an auricular premature systole when $\mathbf{P}_{c}$ is abnormal, and a sinus beat when $P_{c}$ resembles $P_{a}$ and $P_{b}$. In the former case, we would be dealing with a pair of premature systoles, the first, $R_{f}$, a ventricular, and the second, $P_{c} R_{g}$, an auricular. In the latter case, we would be dealing with an interpolated ventricular premature systole, $\mathbf{R}_{\mathbf{f}}$. However, closer examination of the record and its measurements, together with the data obtained in the later records (see below) are opposed to this interpretation. The argument for the alternative interpretation we adopted is along the following lines.

The bizarre $P_{c}$ waves resemble in contour retrograde $P$ waves which are usually small and upright in lead I and sharply inverted in leads II and III. They are therefore considered 
retrograde $\mathrm{P}$ waves. Such retrograde $\mathrm{P}$ waves could be due either $(a)$ to retrograde conduction of the impulse from the ventricular premature systole $R_{\mathrm{f}}$ or $(b)$ to retrograde conduction of an impulse arising in the A-V node which gives rise to $R_{g}$. Since a nodal premature systole, as assumed in $(b)$ which shows fixed coupling with the preceding QRS, is most likely due to re-entry, the alternative explanations for the retrograde $P$ waves become identical.

The acceptance of the view that the bizarre $P$ waves are retrograde in origin permits consideration of the $P_{c}$ intermediate between the bizarre ones and those resembling the sinus $\mathbf{P}$ waves. The intermediate $P_{c}$ waves can be considered to be due to simultaneous invasion of the auricles from the sinus node and by the retrograde impulse. A comparable phenomenon seen in the transition from sinus to A-V nodal rhythm has led to the description of such beats as transitional complexes. (Lewis, 1925), or fusion beats (Katz, 1941). The alternative explanation for the variable $P_{c}$ contour would be to ignore the retrograde contour and conceive the $\mathbf{P}_{c}$ complexes as being auricular premature systoles from varying foci. The latter explanation is not as tenable as the fusion $P$ assumption since it ignores the constancy of $R_{f}-R_{g}$ regardless of the $\mathbf{P}_{c}$ contour and would not explain the occurrence of a $\mathbf{P}_{c}-\mathbf{R}_{\mathrm{g}}$ interval of less than 0.12 sec. in a subsequent record (Fig. $2 \mathrm{~A}$ ).

A

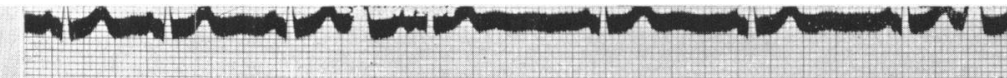

B

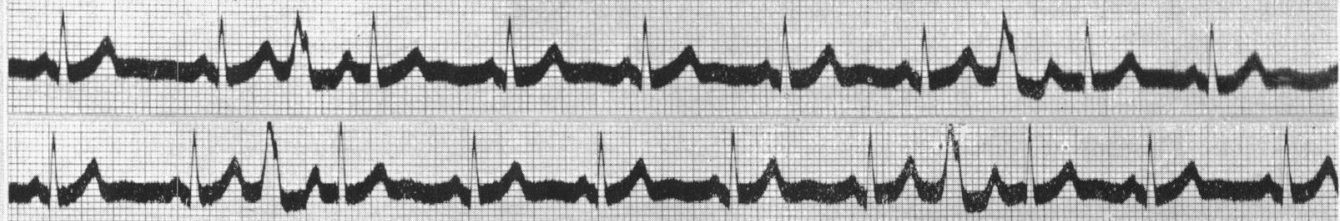

D

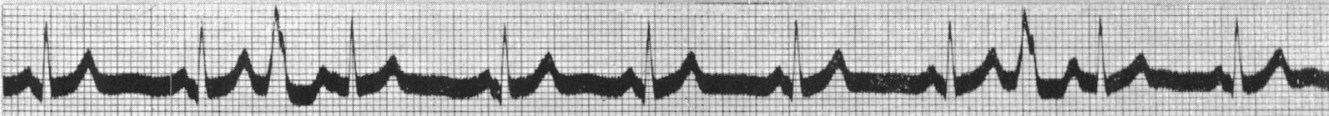

E

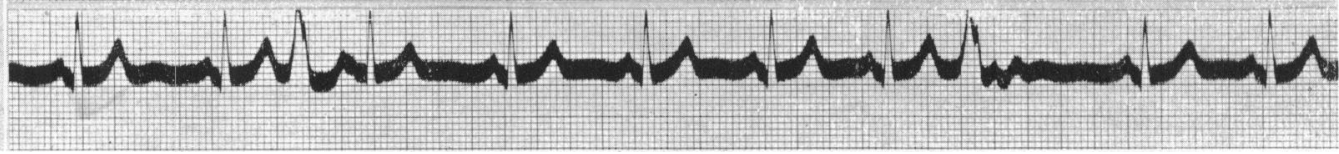

Fig. 2.-Electrocardiograms (lead II) taken one month after that shown in Fig. 1. (A) is a record at rest and shows two instances where $P_{c}-R_{g}$ is less than $0 \cdot 12 \mathrm{sec}$. (B) is a record after exercise, and (C) during left carotid sinus pressure. They show that $R_{\mathbf{f}}-\mathbf{R}_{\mathrm{g}}$ (second pair in each strip) is longer than the $\mathbf{R}_{\mathbf{f}}-\mathbf{R}_{\mathrm{g}}$ interval obtained in records at this time. (D) is also taken during left carotid sinus pressure. $R_{f}-R_{g}$ in this strip, as in the first pair of (B) and (C) and both pairs in (A) show the usual duration found at this time. (E) taken immediately after left carotid sinus pressure shows one $R_{f} \cdot P_{c}$ combination not followed by $R_{g}$. The $P_{c}$ in this instance is clearly of sinus origin. Discussed in text.

If the view that $P_{c}$ waves differing from $P_{a}$ and $P_{b}$ in contour are associated with retrograde conduction, then the $\mathbf{R}_{\mathbf{g}}$ that follows such a $\mathbf{P}_{\mathrm{c}}$ cannot be of sinus node origin, since the sinus node impulse either does not invade the auricles or at least does not reach the A-V junctional tissues. $\mathbf{R}_{\mathbf{g}}$ must therefore be due to the retrograde impulse responsible for $\mathbf{P}_{\mathbf{c}}$ and hence is part of a reciprocal rhythm initiated by the premature ventricular systole $\mathbf{R}_{\mathbf{f}}$.

There remains the group of $\mathbf{P}_{c}$ waves which are almost identical with $\mathbf{P}_{a}$ and $\mathbf{P}_{\mathrm{b}}$. Two alternatives exist for $\mathbf{R}_{\mathbf{g}}$ following such $\mathbf{P}$ waves. In fact both might be operative on different occasions. The simpler explanation would be to assume that $\mathbf{R}_{\mathbf{g}}$ is of sinus node origin. In favour of this view is the fact that in subsequent records there were three occasions on which $\mathbf{R}_{\mathbf{f}}-\mathbf{R}_{\mathrm{g}}$, otherwise remarkably constant, was prolonged compared to the other $\mathbf{R}_{\mathbf{f}}-\mathbf{R}_{\mathbf{g}}$ intervals and associated with a $\mathbf{P}_{c}-P_{g}$ longer than the usual sinus $P-R$, as might be expected after an interpolated ventricular premature systole (e.g. the second group in Fig. 2 B and Fig. 2 C). In 
these three instances $R_{\mathbf{g}}$ is most likely of sinus origin. The remarkable constancy, with the three exceptions noted, of the $R_{f}-R_{g}$ interval (regardless of the position and contour of $P_{c}$ ) favours the idea that $R_{g}$ is part of a reciprocal rhythm. This reciprocal rhythm is initiated by $R_{f}$ and re-entry to the ventricles of the retrograde impulse occurs at a point below the auricles somewhere in the $\mathrm{A}-\mathrm{V}$ junctional tissues above the bifurcation of the common $\mathrm{A}-\mathrm{V}$ bundle at a time before the sinus impulse reaches the re-entry point.

Several other facts became apparent in the series of records (of lead II only) taken a month after Fig. 1 which support our explanation. These were recorded during rest, after exercise, during and after left carotid sinus pressure, and during the first 45 minutes of the action of atropine sulphate (1/75 grain intramuscularly) (Fig. 2).

(1) In these records a total of $91 R_{f}$ complexes were encountered, the $P_{c}$ that followed was of sinus contour in 87, retrograde in none, and suggested a fusion $\mathrm{P}$ in 4 . In 38 instances a combination $\mathbf{R}_{\mathrm{f}} \mathrm{P}_{\mathrm{c}} \mathrm{R}_{\mathrm{g}}$ occurred.

(2) There was a sinus arrhythmia present with slight variations of $\mathrm{P}$ contour indicating a wandering pacemaker, but the $P_{c}$ contour (even allowing for the distortion due to its superposition on the $S-T-T$ of $R_{f}$ ) varied more than the sinus $P$ waves.

(3) There was a shortening of the $\mathrm{P}-\mathrm{P}_{\mathrm{c}}$ interval and a lengthening of the $\mathrm{P}_{\mathrm{c}}-\mathrm{P}$ interval apparently due to the same mechanism as so-called ventriculo-phasic sinus arrhythmia in heart block.

(4) The occurrence of $R_{g}$ depended on the $R_{f}-P_{c}$ interval. $R_{f}-P_{c}$ varied from less than 0.16 to $0.32 \mathrm{sec}$. when $R_{g}$ was absent, and from 0.34 to $0.42 \mathrm{sec}$. when $R_{g}$ followed. The variations in $R_{f}-P_{c}$ were due to the sinus arrhythmia as well as to the occurrence of the premature ventricular systole, $R_{f}$, at different times after the sinus $Q R S$, i.e. $R_{e}-R_{f}$ varied from $0.36-0.56 \mathrm{sec}$.

(5) In all instances where $P_{c}$ was not followed by $R_{g}, P_{b}$ appeared to be of sinus origin.

(6) The interval $R_{f}-R_{g}$ was remarkably constant, being $0.48 \mathrm{sec}$. In three instances it was longer, equalling $0 \cdot 51,0 \cdot 52,0 \cdot 52 \mathrm{sec}$. (Fig. $2 \mathrm{~B} \& \mathrm{C}$ ). In these three, $\mathrm{P}_{\mathrm{c}}$ occurred relatively early (at the shortest $R_{f}-P_{c}$ distance followed by an $R_{g}$ ), resembled the sinus $P$, and was associated with a prolonged $P_{c}-R_{g}$ interval $(0 \cdot 15,0 \cdot 18,0 \cdot 18 \mathrm{sec}$. respectively) as compared with other $P_{c}-R_{g}$ intervals $\left(0.06-0.14\right.$ sec.). These facts confirmed the view that $R_{f} R_{g}$ represent reciprocal rhythm except when the $R_{\mathbf{f}}-R_{g}$ interval is definitely prolonged; in the latter case, $R_{g}$ is considered to be of sinus origin. This has already been referred to above.

(7) The occurrence of $P_{c}-R_{g}$ of less than $0.12 \mathrm{sec}$. was encountered three times (Fig. $2 \mathrm{~A}$ ), indicating that $R_{g}$ in these instances can not be of sinus origin, nor due to any impulse spreading from the auricles to the ventricles after the inscription of $P_{c}$, a matter already referred to above. In determining the position of $P$ in this and other instances, a task not always easy, we made use of the duration of electrical systole of $R_{f}$ in other beats in the record.

The mechanism which we believe underlies this arrhythmia can best be summarized diagrammatically as in Fig. 3. A sinus rhythm is present throughout and in addition there are ventricular premature systoles; these two pacemakers determine the arrhythmia. The ventricular premature beats have retrograde conduction and the contour of $\mathbf{P}_{c}$ following them will depend upon where the retrograde impulse and sinus impulse meet and interfere with each other. This is determined by the relative time of discharge of the sinus and ventricular pacemakers and by the retrograde conduction time. The possibilities are:

(a) near the sinus node (Fig. $3 \mathrm{~A}$ ) in which case the auricles will be controlled by the retrograde impulse and $\mathrm{P}$ will have the retrograde $\mathrm{P}$ contour;

(b) near or within the A-V junctional tissue (Fig. 3, C, D, and E) in which case the auricles will be controlled by the sinus impulse and $\mathrm{P}$.will be of sinus $\mathrm{P}$ contour; or

(c) somewhere in the auricles (Fig. 3 B) in which case the auricles will be controlled in part by the sinus and in part by the retrograde impulse and $P$ will have a contour intermediate between the sinus and retrograde $P$, i.e. it will be a fusion $P$. 


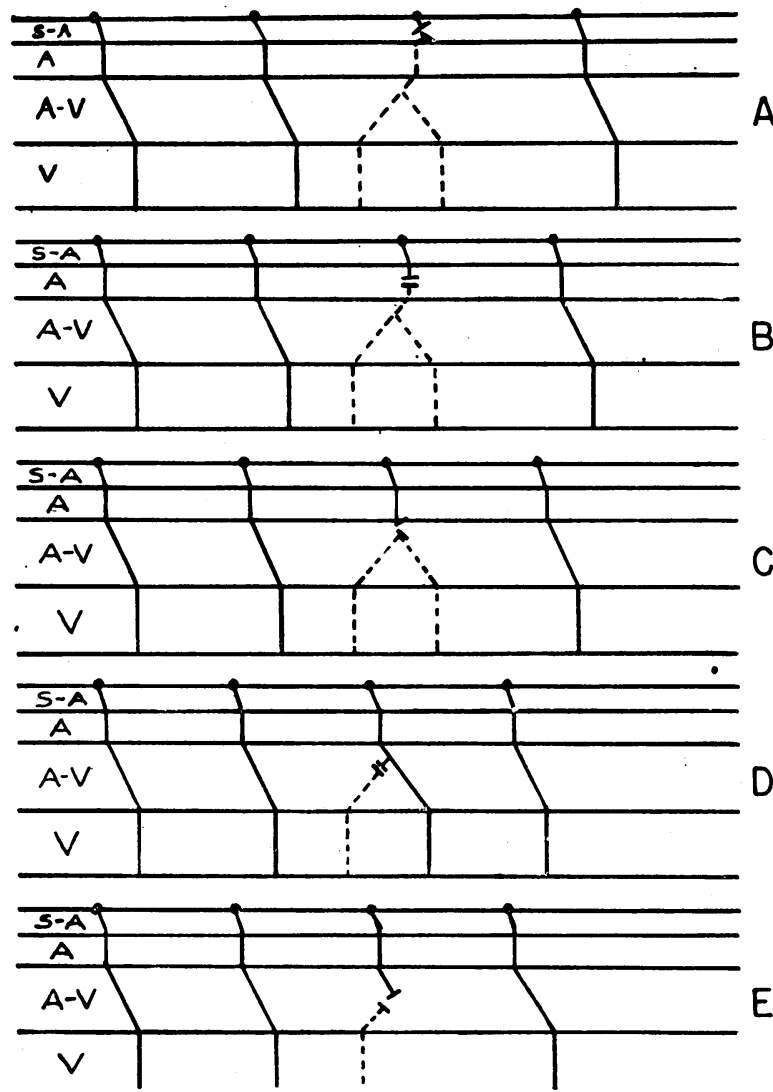

FIG. 3.-A series of diagrams illustrating the various circumstances encountered in this patient, showing the various locations of interference between the sinus and retrograde impulses as well as the conditions under which re-entry and reciprocal rhythm are established. Discussed in text. Dotted lines represent the ventricular premature systole, the retrograde conduction, and the re-entry leading to reciprocal rhythm. $\mathrm{S}-\mathrm{A}, \mathrm{A}, \mathrm{A}-\mathrm{V}$, and $\mathrm{V}$ represent conduction respectively within the sinus node, auricles, A-V junction, and ventricles.

The retrograde impulse in travelling through the A-V junctional tissue finds a point at which it can become antegrade again and re-enter the ventricles. The fact, pointed out above, that the interval $R_{\mathbf{f}}-\mathbf{R}_{\mathrm{g}}$ is fixed except on three occasions begs coincidence too much and suggests that in all but the three exceptions the combination of the premature ventricular systole and the ventricular beat that follows are instances of reciprocal rhythm. Since this occurs at times when the auricles are under the control of the sinus node and not invaded by the retrograde impulse from the premature ventricular systole, and at other times when $\mathbf{P}_{c}$ suggests a fusion $P$, with a $P_{c}-R_{g}$ interval of less than 0.12 sec., it follows that re-entry takes place below the auricles in these cases, and therefore presumably this is the location of reentry in all cases where reciprocal rhythm occurs. Further the fact that the second ventricular complex of the reciprocal rhythm resembles those of sinus origin indicates that the re-entry point is above the bifurcation of the common bundle. It is our inclination to believe that it is somewhere in the A-V node where branching is more prevalent than in the A-V bundle. The conditions for re-entry have been clearly analysed by Schmitt and Erlanger (1928) as a monodromic condition in some fibers with a heterodromic condition in adjacent fibers. In other words different degrees of unidirectional block in adjacent fibres predispose to re-entry of the impulse. In our case the retrograde conduction time (R-P) of about $0.40 \mathrm{sec}$. in those instances where $P_{c}$ is retrograde (Fig. 1), as compared to a $P-R$ of $0.14 \mathrm{sec}$. of the sinus beats proves that retrograde conduction is markedly delayed.

We are now prepared to consider what happens in the A-V junctional tissue in those beats 
in which the sinus impulse invades it after the premature ventricular systole is initiated. Two main possibilities exist.

The sinus impulse can meet the retrograde impulse before the latter has passed the point of re-entry, and so prevent not only the retrograde impulse from entering the auricles but also the establishment of the reciprocal rhythm. If the sinus impulse enters sufficiently early it itself will be prevented from entering the ventricles and the ordinary variety of premature ventricular systole with a fully compensatory pause will ensue (Fig. $3 \mathrm{E}$ ). If, however, the sinus impulse enters later so as to reach the point of re-entry just ahead of the retrograde impulse, it may find the tissue through which the retrograde impulse usually re-enters in a state permitting its passage into the ventricles. Here, then, the re-entry would be prevented but a second ventricular complex would follow the premature ventricular systole at an interval somewhat longer than that between the ventricular beats of reciprocal rhythm, because of prolongation of the conduction through the A-V junction resulting from the fact that the sinus impulse travels through earlier than the re-entry impulse (Fig. $3 \mathrm{D}$ ). This then would be an example of an ordinary interpolated ventricular premature systole.

The sinus impulse can meet the retrograde impulse after the latter has passed the point of re-entry. In this case, the sinus impulse would prevent the retrograde impulse entering the auricles, and the retrograde impulse would prevent the sinus impulse from reaching the ventricles. However, since re-entry had occurred, the retrograde impulse would actually cause the following ventricular beat. In short, the post-extrasystolic beat would have a sinus $\mathbf{P}$ wave and a ventricular complex due to re-entry of the retrograde impulse of the preceding premature ventricular systole, the $P$ and $Q R S$ thus being unrelated (Fig. $3 \mathrm{C}$ ). In these cases the $R-R$ interval between the two beats of the reciprocal rhythm would be expected to have the same duration as in those cases in which a retrograde $P$ can be shown to occur between them; and this was actually true.

The occurrence of re-entry before the impulse reaches the auricles, found in our case, may be found in any case of reciprocal rhythm, as Drury (1924) suggested and as has been postulated by Scherf and Shookhoff (1926) and by Cutts (1937). The case of Gravier et al. (1939) showing reciprocal rhythm and varying $P$ wave contour could be explained, in like manner, by assuming re-entry below the auricles with interference between the retrograde and sinus impulses above the level of re-entry. Obviously, the "sandwiching" of the $P$ wave between two ventricular complexes (White, 1915) does not constitute an integral part of reciprocal rhythm. Even when such a $P$ wave is retrograde in character it indicates merely retrograde conduction, a condition necessary for re-entry.

The significance of a sinus $P$ between a pair of ventricular beats in a case like ours may be evaluated by the duration of the P-R interval compared with the P-R of the sinus beats. When the $P-R$ is equal to or shorter than the sinus $P-R$, the second beat would be considered a reciprocal beat, provided the $R-R$ interval equalled that of known reciprocal beats. When the $\mathbf{P}-\mathbf{R}$ is longer than the sinus $\mathbf{P}-\mathbf{R}$ duration and the $\mathbf{R}-\mathbf{R}$ interval is also prolonged while $\mathbf{P}$ resembles the sinus $P$ waves, then, the second beat would be considered a sinus beat. The fortuitous combination of a sinus $P$ wave between two ventricular beats indicates "pseudoreciprocal rhythm" (Katz and Kaplan, 1938).

The case reported by us suggests that even in the absence of retrograde conduction in the form of retrograde $\mathbf{P}$ waves, re-entry may be responsible for other instances of fixed coupling, whether this occurs as in our case between two premature systoles or between a premature systole and the preceding sinus beat. This possibility though previously considered (Mines, 1913; de Boer, 1921; and Lewis, 1925) has been questioned by others (Scherf, 1930, and Rothberger, 1931). Our case is important since it definitely favours the re-entry mechanism, When the second ventricular complex is bizarre compared with the sinus beats the re-entry may be below the bifurcation of the common bundle, and this variety is the common one encountered. Probably other instances like ours will be discovered. 


\section{SUMMARY AND CONCLUSIONS}

A case is presented showing sinus rhythm and ventricular premature systoles followed at a fixed interval by another premature ventricular complex of supraventricular origin. The occasional presence of inverted $\mathbf{P}$ waves between the two premature ventricular beats suggested reciprocal rhythm. This was substantiated not only by the constant interval between the two premature ventricular beats but also by the occurrence of $\mathbf{P}$ waves preceding the second premature ventricular beat at a distance so short as to preclude the possibility of conduction of an impulse from the auricles to the ventricles in the second beat.

In our case evidence is presented to suggest that the point of re-entry was below the auricles and above the bifurcation of the common bundle, presumably within the A-V node.

It is pointed out that the retrograde $P$ waves in cases of reciprocal rhythm due to re-entry of retrograde impulses merely indicate the presence of retrograde conduction, without in themselves constituting an essential part of the mechanism underlying reciprocal rhythm.

The various possibilities of interference, in our case, between the sinus impulse and retrograde impulses causing reciprocal rhythm are evaluated and instances of each cited.

Our case indicates that instances of fixed coupling of premature ventricular beats even in the absence of $\mathbf{P}$ waves, indicative of retrograde conduction, may be examples of re-entry.

\section{REFERENCES.}

1. Bishop, L. F. (1921). J. Amer. med. Ass., 77, 31.

2. Blumgart, H. L., and Gargill, S. L. (1930). Amer. Heart J., 5, 424.

3. Cutts, F. B. (1937). Ibid., 14, 717.

4. De Boer, S. (1921). Arch. ges. Physiol., 187, 193.

5. Dock, W. (1928). Arch. intern. Med., 41, 745.

6. Drury, A. N. (1924). Heart, 11, 405.

7. Fogelson, L. J. (1929). Z. Kreislaufforsch., 21, 290.

8. Gallavardin, L., and Gravier, L. (1921). Arch. Mal. Coeur., 14, 71.

9. Gravier, L., Froment, R., and Guiran, J. B. (1939). Ibid., 32, 622.

10. Katz, L. N., and Kaplan, L. G. (1938). Amer. Heart J., 16, 694.

11. Katz, L. N. (1941). Electrócardiography, Philadelphia.

12. Korth, C., and Schrumpf, W. (1936). Disch. Arch. klin. Med., 178, 589.

13. Lewis, T. (1925). The Mechanism and Graphic Registration of the Heart Beat, 3rd ed. London.

14. Mines, G. R. (1913). J. Physiol., 46, 349.

15. Reid, W. D. (1930). Amer. Heart J., 5, 524.

16. Rothberger, C. J. (1931). Ergeb. Physiol., 32, 681.

17. Scherf, D., and Shookhoff, Ch. (1926), Wien. Arch. inn. Med., 12, 501.

18. Scherf, D. (1930). Z. ges. exper. Med., 70, 375.

19. - (1941). Arch. intern. Med., 67, 372.

20. Schmitt, F. O., and Erlanger, J. (1928). Amer. J. Physiol., 87, 326.

21. Samojloff, A., and Tschernoff, A. (1930). Z. ges. exper. Med., 71, 768.

22. v. Dobozy, E. (1936). Klin. Wschr., 15, 1160.

23. White, P. D. (1915). Arch. intern. Med., 16, 517.

24. (1921). Ibid., 28, 213.

25. Wolferth, C. C., and McMillan, T. M. (1939). Amer. Heart J., 4, 521. 